\title{
Antenna Optimization by Using NEWUOA
}

\author{
Weixin Zhao*, Arijit De, Daniel García Doñoro, Yu Zhang, Tapan K Sarkar \\ Department of Electrical Engineering and Computer Science, Syracuse University \\ Syracuse, NY 13244-1240, USA. Tel: (315)443-3775 \\ Email: ade@syr.edu
}

\begin{abstract}
The NEWUOA is a newly developed trust region based derivative-free method for solving unconstrained optimization problems. We present in this paper this powerful tool for antenna optimization. Two antenna design examples are optimized using simplex method, conjugate gradient method and the NEWUOA algorithm. Optimization results are presented and compared. The NEWUOA shows good performance and is proved to be a suitable tool for antenna optimization.
\end{abstract}

\section{Introduction:}

Optimization in engineering is the study of complex problems and the procedure to obtain the best solution to it. The best solution could be either the minimum or the maximum value of a predefined objective function. Successful design of a good antenna involves a lot of variables to be optimized. A powerful optimization method is hence essential. In practice, several optimization methods are widely used and many methods were devised for specific situations. Some of the most popular ones are like the Nelder and Mead simplex method (uses simplex method as described below), steepest descent method and the gradient method [1]. Since the evaluation of the cost function for a real antenna problem involves considerable time, choosing the proper initial values of the variables [2] and a fast convergence of the optimization algorithm is an essential feature.

\section{Description of the NEWUOA algorithm:}

The NEWUOA (new unconstrained optimization algorithm) is an unconstrained optimization method by quadratic approximation based derivative-free method [3-4]. So there are no constraints on the variables. As we know, if the gradient to the objective function is available, then first order conditions helps to identify the solution. However, in practice, evaluation of derivatives is not always easily achieved. NEWUOA offers a platform for optimization without the calculation of the derivatives. This merit makes it more widely suitable dealing with practical problems. 
The method seeks to calculate the least value of a function $\mathrm{F}(\mathrm{x})$ by applying the trust region iteration for adjusting the variables. The user needs to provide a starting vector $\mathrm{x}$ of size $\mathrm{n}$, which contains the values of the $\mathrm{n}$ optimization variables, values of the parameters $\rho$ beg and pend, and an integer ' $m$ ' from the interval $[n+2$, $1 / 2(n+1)(n+2)]$. Here $\rho b e g$ and pend are the size of the initial and the final search region around the current value of $\mathrm{x}$, respectively, and pend can be used to control the final accuracy of the desired solution. The procedure of NEWUOA employs a quadratic model (quadratic polynomial approximation to the function $\mathrm{F}$ ) that interpolates just $m$ values of the objective function. The value $m=2 n+1$ is recommended, so that the complexity of the evaluation of the algorithm is of the order of $(m+n) 2$, allowing $n$ to be quite large.

\section{Numerical examples and results}

To illustrate the performance of NEWUOA in antenna optimization, we considered two examples: the Yagi-Uda antenna and the two-dimensional dipole array. First let's consider the design of 6-element Yagi-Uda antenna with one reflector, a driven element and 4 directors as shown in Fig. 1. For simplicity, the Yagi antenna is only optimized for the maximal forward gain at the frequency of $300 \mathrm{MHz}$. We set all reflectors have the same length and spacing. So there are six parameters to be optimized: the length of the reflector $l_{\text {ref, }}$, the length of the driven element $l_{\text {driv }}$, the length of all directors $l_{\mathrm{dir}}$, the spacing between the reflector and the driven element $S_{\text {ref, }}$, the spacing between driven element and the first director $S_{\text {driv, }}$ and the spacing between two adjacent directors $S_{\text {dir }}$. The spacing is allowed to vary between $0.1 \lambda$ and $0.4 \lambda$, and the length is allowed to vary between $0.2 \lambda$ and $0.8 \lambda$. For the unconstrained method NEWUOA, we can arbitrarily set the length/spacing to be the boundary values if the length/spacing goes beyond the boundary during the optimization.

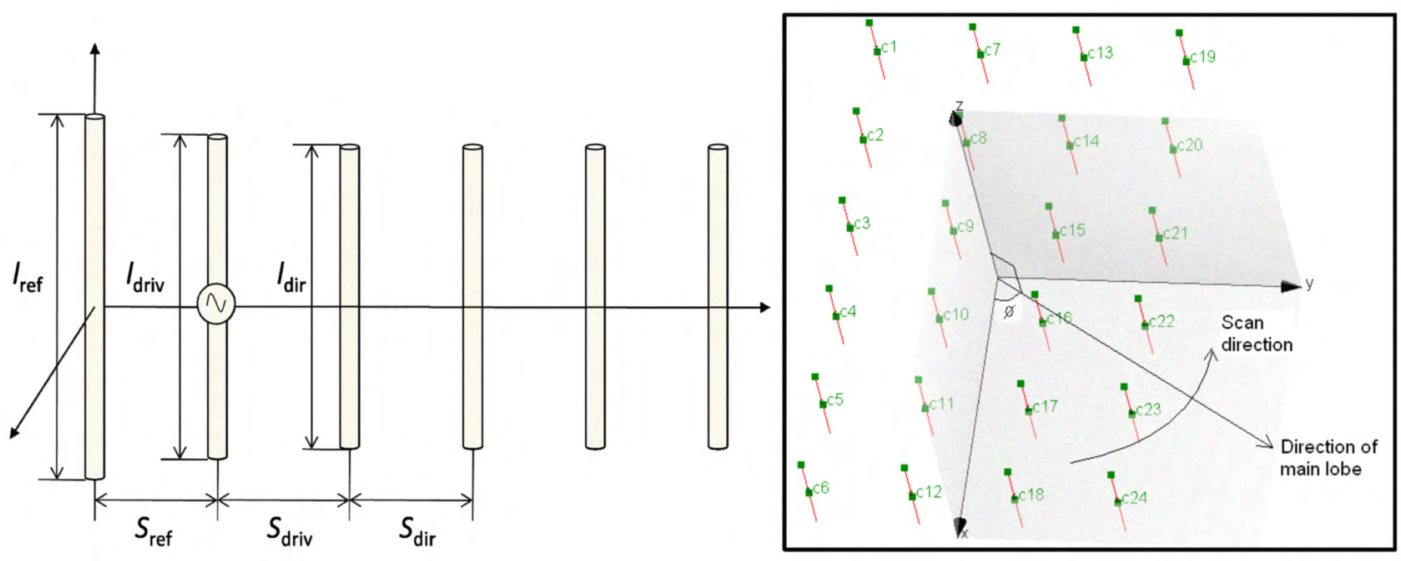

Fig. 1 A 6-element Yagi-Uda antenna array

Fig. 3 Structure of the $4 \times 6$ dipole 
The NEWUOA optimization algorithms are incorporated in a commercially available frequency domain integral equation based electromagnetic solver, TIDES [5]. It is then compared with the simplex and the gradient based algorithm. The initial value for every variable is set to be $0.25 \lambda$. The convergence of each of the algorithm is shown in Fig. 2(a) below.

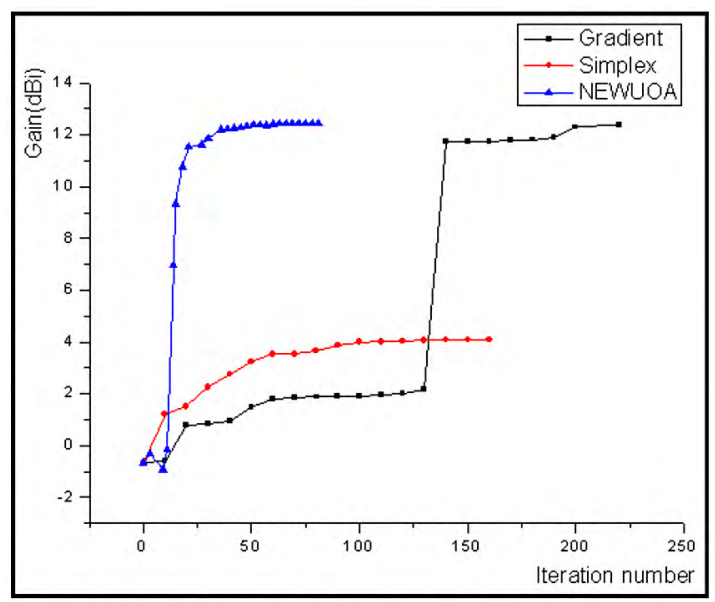

(a) 6-element Yagi-Uda array

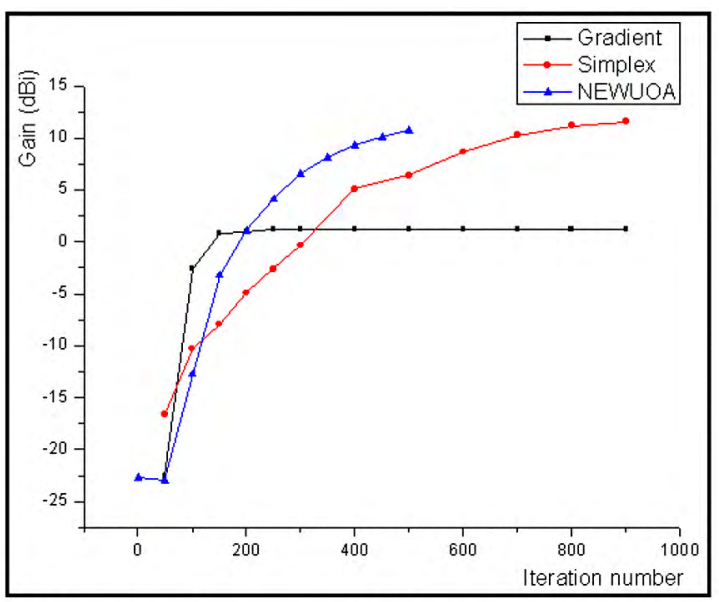

(b) two-dimensional dipole array

Fig. 2 Comparison of the convergence of the optimization algorithms for two antenna

From the result shown above, it's easy to find that NEWUOA achieves convergence after around 40 iterations (function evaluations) and the 6 element yagi antenna has a gain of around $12.45 \mathrm{dBi}$. The conjugate gradient method achieves convergence after about 140 iterations and achieves a gain comparable to the result of NEWUOA algorithm. The simplex method on the other hand gets stuck in some local optima and doesn't achieve a good gain, though it has a tendency to converge faster than the other two methods. We further mention that the initial values chosen for this problem is not a good one, as the gain based on the starting value is below $0 \mathrm{dBi}$. A better choice of the initial value might yield different convergence characteristics and higher gain. But from our experiment we found the trend to remain almost similar whereby the NEWUOA is considerably better than the other algorithms. Using the above initial values and the local optimization algorithms, the highest gain (best results) obtained in the yagi case is within $1 \mathrm{~dB}$ within the published result [6].

The second optimization example is a 4 by 6 two-dimensional dipole array as shown in Fig. 3. Each dipole is half-wavelength long and is loaded with $50 \mathrm{ohms}$ at the center. The space between two adjacent dipoles is also half-wavelength. Our objective is to optimize the voltage excitations of each dipole element in the array, in order to obtain maximum gain in the end-fire direction, at the angle $\varphi=45^{\circ}$. We run our simulation at the frequency of $430 \mathrm{MHz}$. For the initial value, we set the voltage 
excitations on each antenna element as 1 volt. The optimization result is shown in Fig. 2(b) above.

The gradient method doesn't achieve good result in this example, while the simplex method converges to the highest gain of about $11.60 \mathrm{dBi}$ but takes 900 iterations, which is nearly double the amount of iteration used by NEWUOA which converges to a similar value closer to it within a $\mathrm{dBi}$. We find that choosing a better initial value like as described in [2], helps to obtain better gain by the optimization algorithms.

\section{Conclusion}

The antenna design and its variable optimization is a highly complicated and time consuming job. The three optimization methods used above are tested on two antenna design examples. It is difficult to judge the best algorithm based on such limited examples presented above. But we can claim that the NEWUOA showed better stability for its performance and achieved relatively better results within a few iterations, for several classes of problems that we have tested. However, the main disadvantage for these three methods is that they are highly dependent of the initial value for obtaining the best optimization results.

\section{Reference}

[1] William H. Press, 'Numerical Recipes in Fortran', Cambridge University Press, 1992, 2nd edition

[2] Weixin Zhao, Arijit De, Yu Zhang, Xiaomin Lin, Tapan K Sarkar, 'Optimization of the End-fire Beam Pattern of Two-Dimensional Dipole Array', IEEE AP-S International Symposium, San Diego, CA, July 2008.

[3] M. J. D. Powell, "The NEWUOA software for unconstrained optimization without derivatives", in Large-Scale Nonlinear Optimization, eds. G. Di Pillo and M. Roma, Springer (New York), pp. 255-297.

[4] M. J. D. Powell. "Developments of NEWUOA for unconstrained minimization without derivatives", Technical Report DAMTP 2007/NA05, Department of Applied Mathematics and Theoretical Physics, Cambridge University, UK, 2007.

[5] Y. Zhang, T. K. Sarkar, H. Moon, A. De, and M. C. Taylor, "Solution of large complex problems in computational electromagnetic using higher order basis in MoM with parallel solvers," in Proc. IEEE Antenna and Propagation Symp., Jun. 9-15, 2007, pp. 5620-5623.

[6] D. K. Cheng, "Gain optimization for Yagi-Uda arrays," IEEE Antennas Propagat. Mag., vol. 33, pp. 42-45, June 1991. 